\title{
Median Door to Needle Antibiotic in Potential Febrile Neutropenic Patients in an Emergency Department of a University Metropolitan Tertiary Hospital in Barcelona (Spain): Results of an Initial Audit
}

\author{
Blanco MC* \\ Nefro-Urological Cancer Nurse Navigator Hospital Clinic \\ de Barcelona, Spain \\ *Correspondling author: Meritxell Casanovas Blanco, \\ Nefro-Urological Cancer Nurse Navigator Hospital Clinic \\ de Barcelona, C/Villarroel, 17008036 Barcelona, Spain \\ Received: February 12, 2021; Accepted: March 03, \\ 2021; Published: March 10, 2021
}

\begin{abstract}
Objective: Febrile Neutropenia is a potentially life-threatening complication, which can increase morbidity and poorer clinical outcomes when first antibiotic dose is delayed in patients with potential Febrile Neutropenia (FN). Lately, is remarkably gaining consideration within the experts, aware of the prevailing poor FN guidelines compliance as reported in written literature. This research explores the ED adherence to Key Performance Indicators of ED care as indicated in evidence-based guidelines in a tertiary University Hospital in Barcelona.

Methods: This is a retrospective observational cohort study of oncology patients receiving anticancer therapy in the previous 30 days of presenting to ED with: non-specific neutropenia, non-specific bacteraemia, fever, infection or septicaemia, from the $1^{\text {st }}$ January 2017 until the $31^{\text {st }}$ of December 2017. 166 patients were elective for the study.

Results: Time to first $A B$ dose within one hour only occurred in $16(9.6 \%)$ of the cases. $69.3 \%$ (115) of the patients received their first $A B$ dose within more than two hours which is non-complaint with the FN protocol.

Conclusions: Evidence of some poorer outcomes accentuate an impending need to take action (implement policies, establish clinical pathways and an effective model of care) to improve FN ED management and achieve better clinical results.

Keywords: Emergency service; Antineoplastic agents; Cancer; Therapy associated adverse effects; Febrile neutropenia; Guidelines; Antibiotic
\end{abstract}

\section{Introduction}

Febrile Neutropenia (FN) is remarkably gaining consideration within the experts of the oncology field, aware of the prevailing poor FN guidelines compliance as reported in written literature. The author herself, having critically evaluated the international research in regard to Emergency Department (ED) FN protocol compliance and also due to clinical practice experience, totally endorses the poor compliance reported $[1,2]$. Despite the potential negative clinical outcomes and the economic burden, a delay of Antibiotic (AB) treatment places to an already strained healthcare system [3], an optimum venue or an effective strategy to manage this population has not yet been identified.

Oncology patients undergoing anticancer therapy present frequently to $\mathrm{ED}$ with potential $\mathrm{FN}$ as a side effect of the treatment. A study conducted in a UK regional cancer centre reported a FN annual incidence of 19.4 per 1000 oncology admissions [4]. Febrile Neutropenia is a potentially life-threatening complication and can increase morbidity and poorer clinical outcomes when the first $A B$ dose is delayed in patients receiving anticancer agents due to potential immunosuppression [5].
The variable known as 'Door-To-Needle Time' (DTN) is defined as the time between patient's arrival at the ED triage and the administration of first $A B$ dose [6]. In Spain, guidelines for a DNT do not exist. However, in our hospital, FN in oncology patients undergoing anticancer therapy is a paramount concern and, therapeutic education to present immediately to the $\mathrm{ED}$ in case of experiencing a temperature of $38^{\circ} \mathrm{C}$ and above for physical assessment and blood cultures plus $\mathrm{AB}$ administration, is strongly highlighted.

The author's work setting is a tertiary University hospital in Barcelona with a foreseen ED renovation to allocate ten beds dedicated only to oncology emergencies. This study was designed to audit ED compliance with FN protocol in the oncology population undergoing anticancer therapy to generate crucial clinical and health system delivery data, to optimize the ED model of care for the target population. Our main objective was to explore the ED adherence to Key Performance Indicators (KPI) of ED care as indicated in evidence-based guidelines. This is the first research study lead by nurses in regard to DTN, to our knowledge, in Spain.

\section{Subjects and Methods}

This was a retrospective observational cohort study of oncology 
patients who received anticancer therapy in the previous 30 days of presenting to ED with a diagnosis of: non-specific neutropenia, non-specific bacteraemia, fever, infection or septicaemia, from the $1^{\text {st }}$ January 2017 until the $31^{\text {st }}$ of December 2017. The study setting was a major tertiary metropolitan teaching hospital and Level I Trauma Centre, with an annual ED attendance rate of 130.000 visits in Barcelona.

Records were retrieved from routinely collected administrative data, which capture all visits to the ED (data source: SAP). The Chemotherapy Day Unit (CDU) electronic booking system (Genomi) is used to document patient's anticancer regime and date of administration. The following matching procedure was conducted in order to link visits from CDU to ED attendances during the following 30 days of receiving treatment. The variables of 'Arrival date' (ED data set) and 'Presentation date' (CDU data set) were compared in variable 'If $<=30$ days_singleline' to select the presentations inside the 30 -day time frame. If the result was $<=30$ days, they were also included. The matched cases were selected and taken to a new excel sheet where data were checked for errors and assessed for normality in Microsoft Excel. There were no errors identified. Given the large amount of data no normality tests were run (Central limit theorem). A total 166 patients were elective for the study.

The variables collected to analyse were: ID Hospital number, ED attention identification number, date and time of $\mathrm{ED}$ presentation, triage code, time to $\mathrm{AB}$ administration, $\mathrm{AB}$ administered, $\mathrm{ED}$ Length of Stay (LOS), ward of admission and ED discharged diagnosis for all patients. Finally, last date of anticancer agent received, regime received, and oncology diagnosis was captured from GENOMI electronical data.

\section{Statistical analysis}

Every emergency episode was identified using the ED attendance identification number. A descriptive statistical analysis framework (percentages, medians, means, range and interquartile range) and Pearson's chi square test of good fit was chosen to assess the distributional characteristics, identify missing data points and determine statistical significance. Counts and percentages were utilised for dichotomous and categorical variables.

\section{Results}

\section{Clinical characteristics study sample ( $N=166$ episodes)}

There was a higher utilisation of ED by patients diagnosed with solid tumours than haematological patients. Further studies should be conducted with patients with solid tumours and haematology patients to understand the reasons why oncology disease demands more ED attention or the reasons why haematology patients even though having acute complications utilise the ED less (a possibility exists that, given their immune vulnerability, they are directly admitted to the CDU or to the ward) (Table 1).

In terms of clinical characteristics in the study sample 38 of the patients (22.9\%) who attended ED due to potential FN were diagnosed with gastric cancer followed by Genitourinary (GU) cancer patients (29/17.5\%), breast cancer patients (29/17.5\%) and lung cancer patients $(25 / 15.1 \%)$. In regard to presenting complaint, most of the patients (126/75.9\%) were allocated in the category of infection, specific and only (29/17.5\%). Had an immediate diagnose
Table 1: Patient classification according the family cancer type (ONC or HEM). Mean (SD)

\begin{tabular}{|l|l|l|}
\hline Unit & \multicolumn{2}{|l|}{} \\
\hline Haematology & 4 & 2.4 \\
\hline Oncology & 156 & 94 \\
\hline Unknown & 6 & 3.6 \\
\hline
\end{tabular}

Table 2: Diagnosis classification.

\begin{tabular}{|l|c|c|}
\hline \multicolumn{1}{|c|}{ Diagnosis } & Count & $\%$ \\
\hline Gastric Cancer & 38 & 22.9 \\
\hline GU Cancer & 29 & 17.5 \\
\hline Breast Cancer & 29 & 17.5 \\
\hline Lung Cancer & 25 & 15.1 \\
\hline Melanoma & 12 & 7.2 \\
\hline ORL & 8 & 4.8 \\
\hline Brain Cancer & 7 & 4.2 \\
\hline Sarcoma & 4 & 2.4 \\
\hline Obstetric Cancer & 4 & 2.4 \\
\hline Lymphoma & 2 & 1.2 \\
\hline Leukaemia & 2 & 1.2 \\
\hline Unknown & 6 & 3.6 \\
\hline
\end{tabular}

Table 3: Presenting complaint to ED of the Study Sample ( $\mathrm{N}=166$ episodes).

\begin{tabular}{|l|c|c|}
\hline \multicolumn{1}{|c|}{ Presenting complaint } & Count & $\%$ \\
\hline Infection, specific & 126 & 75.9 \\
\hline Neutropenia & 29 & 17.5 \\
\hline Septicaemia & 6 & 3.6 \\
\hline Infection, viral & 3 & 1.8 \\
\hline Infection, non-specific & 2 & 1.2 \\
\hline
\end{tabular}

Table 4: ED triage classification according to MAT model.

\begin{tabular}{|l|c|c|}
\hline \multicolumn{1}{|c|}{ Triage category } & Count & $\%$ \\
\hline Level 2 & 26 & 15.7 \\
\hline Level 3 & 139 & 83.7 \\
\hline Level 4 & 1 & 0.6 \\
\hline
\end{tabular}

Table 5: Door to Needle Time.

\begin{tabular}{|l|c|c|}
\hline \multicolumn{1}{|c|}{ Time from triage to AB } & Count & $\%$ \\
\hline Within 60 mins & 16 & 9.6 \\
\hline Within 120 mins & 15 & 9 \\
\hline More than 120 mins & 115 & 69.3 \\
\hline Missing Data & 20 & 12 \\
\hline
\end{tabular}

of neutropenia. A total of 6 patients, $3.6 \%$ of the sample, presented in ED with septic shock symptoms (Tables 2\&3).

\section{Quality indicators of the study sample ( $\mathrm{N}=166$ episodes)}

Notwithstanding the clinical importance of time to $A B$ commencement, only 26 patients $(15.7 \%)$ were selected as level 2-triage category. The Triage Category Scale used in this particular hospital is the Modelo Triage Andorrano (MAT), which establishes 
for level 2 an immediate nursing attention and a frame-time of 7 minutes for medical attention. The rest of the patients were appointed to a level $3(139 / 83.9 \%)$ and level $4(1 / 0.6)$, nursing attention in these levels can be delayed 15 minutes and more (Table 4).

Time to first $\mathrm{AB}$ dose in the study sample within one hour only occurred in 16 (9.6\%) of the cases. The 69.3\% (115) of the patients received their first $\mathrm{AB}$ dose within more than two hours which is noncomplaint with the FN protocol (Table 5).

\section{Discussion}

One of the most common and severe complication experienced by patients undergoing anticancer therapy is FN. Even though bacteremia is only reported for the $15-20 \%$ of neutropenic patients, it accounts as a major cause of death for the target population [7]. Despite the importance of rapid $\mathrm{AB}$ administration, few oncology studies have addressed timelines of $\mathrm{AB}$ administration during a potential FN episode.

Emergency department management of FN concerns arose in 2008 in the UK when the National Confidential Enquire into Patient Outcome and Death (NCEPOD) critically reviewed cases of patients who died after 30 days of receiving a dose of anticancer agents. The National Chemotherapy Advisory Group (NCAG) reiterated these concerns in 2010. The consequence of little research in this area is a large variation of evidence-based guidelines not based on evidence for FN management on an international level and critically, poor clinical outcomes. The 2010-2011 Australian consensus guidelines for FN management [8] and the National Institute for Health and Care Excellence (NICE) Clinical Guideline 151 issued in the UK in September 2012 [9], emerged as a response to the lack of consensus guiding practice for FN. In 2016, the National Comprehensive Cancer Network (NCCN) stated as paramount in their protocol, starting first $\mathrm{AB}$ dose for patients with $\mathrm{FN}$ within an hour of $\mathrm{ED}$ triage presentation.

The outcomes of the present study show that clinical practice adherence to the guideline was only achieved in the management of $9.64 \%$ of the patients, this finding should be considered within the context of the lack of an FN guideline in the ED setting. Many previous studies concur on our findings of poor adherence with evidence-based guidelines and try a variety of strategies to reduce the DTN time.

Keng et al. described a prospective study implementing a Febrile Neutropenic Pathway (FNP) which incorporated a triage reclassification of FN to the same level as myocardial infarction, private waiting rooms for potential FN patients, a FN alert card, a FN order set, a protocol for $\mathrm{AB}$ administration before confirmation of neutropenia, and FNP training for the ED staff achieving a median time of $81 \mathrm{~min}$ in the adult population [10]. Lim C. and colleagues in their study after implementing an electronic clinical guideline to manage 76 episodes of FN, achieved a median time to $\mathrm{AB}$ first dose administration of 3.9 hours [11]. Hawley retrospectively reviewed 42 charts of FN patients who presented to ED and reported a median time of 138 minutes from door-to-antibiotic [12]. In his article published on 2010, André and his group studied 189 patients with FN at ED presentation and reported that only 19 of them received $A B$ within 90 minutes [13]. And Oakley reported a median time of 135 minutes (range 15-550 minutes) to first $\mathrm{AB}$ dose administration in 71 episodes [14].

\section{Limitations of the Study}

The major strength of this study is the large amount of data collected. It is also one of the firsts nurse-lead studies focused on time to first $\mathrm{AB}$ dose. Considering nurses have a wealth of knowledge about anticancer therapy side-effects and their management [15], and latest policies such as the Department of Health policy (DOH) in the UK enhance the role of oncology nurses in the assessment and management of the cancer patient undergoing anticancer agent therapy [16]; the nursing lead in the management of anticancer therapy complications research will add value to the nurses' new role. The study sets the foundations for future studies to deepen the understanding of current ED management of patients presenting with $\mathrm{FN}$ and to examine novel strategies to address pressures in $\mathrm{ED}$ settings.

One of the limitations of the study is its retrospective nature. Another acknowledged limitation is that unplanned ED presentations were only collected from the study hospital; a possibility exists that a patient receiving anticancer therapy in the Cancer Day Unit (CDU) of the study hospital itself, could have attended the ED of another hospital in the event of a complication. It is therefore possible that some cases were missed in this data set. The study sample was drawn only from one institution and although the sample was big enough to meaningfully represent the hospital study site's population, caution is needed about extrapolating the conclusions beyond the study hospital.

\section{Implication for Clinical Practice}

Evidence of some poorer outcomes accentuate an impending need to take action (implement policies, establish clinical pathways and an effective model of care) to improve FN ED management to achieve better clinical results in the target population. Further work is warranted to achieve best quality of care and better clinical results for cancer patients presenting to an ED with a potential diagnose of FN.

Nurses are key personnel to manage anticancer agent side effects and lead new strategies. At the start of any anticancer agent treatment nurses should assess the patient fitness and risk of suffering complications, specifically febrile neutropenia. Ultimately, it is of upmost importance to educate the patient in preventing and selfmanaging anticancer agent complications, and to rapidly seek for clinical assessment in case of presenting fever.

\section{References}

1. Casanovas-Blanco M, Serrahima-Mackay A. Febrile neutropenia management in cancer patients receiving anti-cancer agents' treatment: Deepening the search to offer the best care. A critical review follow-up. Critical Reviews in Oncology/Hematology. 2020; 153: 103042.

2. Casanovas Blanco M. Critical review of emergency department management of chemotherapy complications in cancer patients. European Journal of Cancer Care. 2019; 28: e12974.

3. NCEPOD-Systemic Anti-Cancer Therapy: For better for worse? (2008). Report. (n.d.). 2021.

4. Schelenz S, Giles D, Abdallah S. Epidemiology, management and economic impact of febrile neutropenia in oncology patients receiving routine care at a regional UK cancer centre. Annals of Oncology. 2012; 23: 1889-1893. 
5. Perron T, Emara M, Ahmed S. Time to antibiotics and outcomes in cance patients with febrile neutropenia. BMC Health Services Research. 2014; 14.

6. Ali N, Baqir M, Hamid A, Khurshid M. Febrile neutropenia: Median door-toneedle time-Results of an initial audit. Hematology. 2015; 20: 26-30.

7. Naeem D, Alshamrani M, Aseeri M, Khan M. Prescribing Empiric Antibiotics for Febrile Neutropenia: Compliance with Institutional Febrile Neutropenia Guidelines. Pharmacy. 2018; 6: 83.

8. Lingaratnam S, Slavin MA, Koczwara B, Seymour JF, Szer J, Underhill C, et al. Introduction to the Australian consensus guidelines for the management of neutropenic fever in adult cancer patients, 2010/2011. Internal Medicine Journal. 2011; 41: 75-81.

9. Bate J, Gibson F, Johnson E, Selwood K, Skinner R, Chisholm J. Neutropenic sepsis: Prevention and management of neutropenic sepsis in cancer patients (NICE Clinical Guideline CG151). In Archives of Disease in Childhood: Education and Practice Edition. Royal College of Paediatrics and Child Health. 2013; 98: 73-75.

10. Keng MK, Thallner EA, Elson P, Ajon C, Sekeres J, Wenzell CM, et al Reducing Time to Antibiotic Administration for Febrile Neutropenia in the Emergency Department. Journal of Oncology Practice. 2015; 11: 450-455.
11. Lim C, Bawden J, Wing A, Villa-Roel C, Meurer DP, Bullard MJ, et al. Febrile neutropenia in EDs: the role of an electronic clinical practice guideline. The American Journal of Emergency Medicine. 2012; 30: 5-11.e5.

12. Hawley EL, Loney M, Wiece M. Development of Tools and Processes to Improve Treatment Times in Patients with Febrile Neutropenia. Clinical Journal of Oncology Nursing. 2011; 15: E53-E57.

13. Andre S, Taboulet P, Elie C, Milpied N, Nahon M, Kierzek G, et al. Febrile neutropenia in French emergency departments: Results of a prospective multicentre survey. Critical Care. 2010; 14: 1-11.

14. Oakley C, Hill A, Chowdhury S. Benefits of a network approach to managing neutropenic sepsis. Cancer Nursing Practice. 2010; 9: 17-21.

15. Farrell C, Lennan E. Nurse-led chemotherapy clinics: issues for the prescriber Nurse Prescribing. 2013; 11: 353-358.

16. Roe $\mathrm{H}$, Lennan $\mathrm{E}$. Role of nurses in the assessment and management of chemotherapy-related side effects in cancer patients. Nursing: Research and Reviews. 2014; 4: 103-115. 